\title{
LETTER TO EDITOR Interdisciplinary evaluation: interface between psychiatry and forensic psychology
}

\author{
Avaliação interdisciplinar: interface entre psiquiatria e psicologia forense \\ Antonio de Pádua Serafim?', Daniel Martins de Barros²
}

\section{DEAR EDITOR}

The study of the causes and consequences of the growing urban violence, the enhancing rates of work disability and labor accidents regarding mental illness, just to mention few examples, increasingly requires the engagement of psychiatrists and psychologists to help enlightening the facts, as answering the questions concerning mental health and justice altogether demands a multilayer comprehension?.

The advances in psychiatry, psychology and more recently in neuropsychology, contributed a great deal for the justice system to interact with these fields of knowledge when dealing in causes regarding the human being, thus richly valuing the role of forensic sciences ${ }^{2}$. Although psychiatry and psychology are different in many ways, in the forensic context it is central that they can work together, as the collaboration among the many mental health professionals enhances the reliability of the documents, lending credibility to the justice decisions ${ }^{3}$.

It is usual that the requiring authority asks for a "psychological and psychiatric evaluation", as if they were a synonymous. As this is frequent, we started the interdisciplinary evaluation in the Forensic Unit of the Instituto de Psiquiatria do Hospital das Clínicas da USP, including, beyond psychiatric and psychological assessment, the neuropsychological and personality evaluation, when applicable. From February/2012 until August/2013 we received 352 justice requirements, $62 \%$ from criminal justice and $38 \%$ from labor or civil prosecutions. The interdisciplinary evaluation was required in $22 \%$ in the first and $43 \%$ of the former.

These data show that the justice system is frequently dealing with questions that cannot be answered without the assistance of a multidisciplinary staff, which we see as an opportunity to expand the boundaries of the mere description of signs and symptoms and help the justice with a comprehensive assessment of the mental state of the individual in legal cases ${ }^{4}$.

Thus the collaboration between these two professions has the potential to increase both the credibility and utility of forensic services to the courts 5 .

\section{DISCLOSURE}

The authors report no conflicts of interest.

1 University of São Paulo, Faculty of Medicine, Hospital das Clínicas (HCFMUSP), Institute of Psychiatry. Methodist University of Sao Paulo, Program of Post-Graduate in Health Psychology. 2 HCFMUSP, Institute of Psychiatry.

Correspondence address to: Antonio de Pádua Serafim Rua Dr. Ovídio Pires de Campos, 785 - Cerqueira César 05403-903 - São Paulo, SP, Brazil

E-mail: a.serafim@hc.fm.usp.br 


\section{REFERENCES}

1. Grisso T, Vincent GM. The empirical limits of forensic mental health assessment. Law Hum Behav. 2005;29(1):1-5.

2. Heilbrun K, Marczyk GR, DeMatteo D, Zilmer EA, Harris J, Jennings T. Principles of forensic mental health assessment: implications for neuropsychological assessment in forensic contexts. Assessment. 2003;10(4):329-43.
3. Gbadebo-Goyea EA, Akpudo H, Jackson CD, Wassef T, Baker NC, Cunningham-Burley R, et al. Collaboration: The Paradigm of Practice Approach between the Forensic Psychiatrist and the Forensic Psychologist. Front Psychiatry. 2012;3:89.

4. Kaufmann PM. Protecting raw data and psychological tests from wrongful disclosure: a primer on the law and other persuasive strategies. Clin Neuropsychol. 2009;23(7):1130-59.

5. Gierowski JK. Complex expertise on the psychiatric health of a criminal. Psychiatr Pol. 2006;40(1):5-17. 